\title{
Design and Development of an Electronic Sieving for Sand Separation using Node MCU System
}

\author{
Pradeep Kumar Krishnan* and Bushra Zaid Humaid Alrisi
}

Mechanical \& Industrial Engineering Department, National University of Science and Technology, Oman

\begin{abstract}
Sand sieving is now considered one of the essential needs in the construction industry. Where businesses collaborate to find the best and highest-quality methods for extracting pure sand suitable for construction. These businesses always require high-quality machines to complete the process flawlessly. This is also to prove its market power and guarantee its products. This research talks about the various mechanisms for designing and manufacturing sand sieving. The sand sieving process expresses the filtering of sand from the rest of the components such as stones or gravel. The literature department studied ten different studies in the design and manufacture of sand sieving machine in different ways. Where these methods vary between using the engine and electricity and using the primitive manual method. After performing these machines several tests and evaluation of the process, it was found that the engine speed affected the energy consumed to sift the sand. Also, the sieve holes are affected by the size of the sifted sand. Where sieves are manufactured in different sizes to suit the size of the sand to be purified. On the other hand, this article contains the future recommendation of the machine to avoid errors and give effective results as needed
\end{abstract}

Keywords: Sieving, Sand Separation, NodeMCU System.

\section{INTRODUCTION}

Construction manufacture is one of the major industries in the world, and sand is an essential contributor to everything regarding construction substances. Where globally, per year more than 50 billion tons of sand are utilized in construction. Therefore, sand has become a valuable resource and increasingly important through the years as the need for it in the international construction industry grows and its availability retreats [1]. In general, sand in construction must be free of gravel and other impurities and clean to guarantee there is no surplus silt, dust, and clay. Moreover, if sand is blended with other substances, it is being eroded readily and demands replacement sooner. So, construction goodness depends significantly on the goodness and pureness of the sand being utilized [2]. In fact, sand is a kind of naturally happening substance that is of a grainy, loose, and segmented configuration which is made up of fine particles like coral, rock, shells, and others [3]. Usually, sand is finer than stone but rough than alluvium. Sand is considered the main component for making mortar, concrete, and plaster [4]. Hence, the sand guarantees there is less opportunity for crumbling and cracking because the goodness of concrete and cement depends on the goodness of the sand utilized in the blend [2]. Commonly sand utilizes in construction, overwhelmingly offers strength, bulk, and *Address correspondence to this author at Mechanical \& Industrial Engineering
Department, National University of Science and Technology, Oman;

Tel: (+968)22347146; E-mail: pradeep.sonatech@gmail.com fastness to other substances like render, cement, asphalt, concrete, mortar, and screed. In addition to that, it utilizes as a basis stratum known as 'blinding' which is put over the stratum of hardcore to offer a level, dry, and clean roof for construction works [4]. In construction, any type of sand cannot be used because the type, source, and goodness of the sand are all very important. For example, sand extracted from a lake is good because it has been washed of impurities. Besides that, if sand is not pure, it may impact the suitable interconnectedness between the sand and the cement. Hence, the result in construction will not be as powerful and sturdy. As well, in the condition of changes in temperature and humidity, concrete may shrink and expand. So, the right blend of sand, cement, and water should be practiced [5]. In the years to come Oman's construction sector is predicted to extend, where the government is focused on infrastructure exploitation as an instrument to ease economic diversification and increasing structural need in the country's ports, power, and water segments. Furthermore, this study aimed to design and manufacture electronic sieving for sand separation machine using the NodeMCU system. Where sieving is a segregation technique according to the variation in particle size [6]. The sieve is a part that accountable for retaining the bigger particles [7]. It allows a small amount of substance to pass through it depending on the size. Hence, the form of the sieve has different sizes to be suitable for the substance to flow [8]. The system of this machine is in line with the modern developments of technology. Therefore, the machine uses advanced systems that are adapted to the 
weather conditions, such as humidity and temperature. This machine is specially designed to reduce energy consumption and make sand sieving easier for workers. The machine is fully controlled by Blynk software, which is directly connected with the NodeMCU. The Blynk software controls the motor through different speeds. As well, it shows the humidity and temperature in the site. Generally, the machine moves in a horizontal movement to cover a big amount of sand [2]. It used a DC motor in the operation due to its feature of rotation motion and converting the energy to motion. Besides that, the innovative part of the machine is the use of a light sensor to cut the laser when the sand passes and starts the motor directly for the sieving process. Actually, this machine is useful for the construction field because of its high efficiency and its unique features. This particular study is important because the existing method of manual sieving involves a lot of time and effort [9]. It also requires manual labor and physical exertion to prepare the sand, limiting those who could perform these tasks. While our prototype only requires one finger to control the software and one person to monitor the operation and bringing the sand.

\section{DESIGN AND ANALYSIS}

\subsection{Design of the System}

This study uses NodeMCU as a control panel of the system. Figure 1 shows the block diagram of the NodeMCU. The panel operates by using a power supply of $5 \mathrm{v}$. As with any control panel, the NodeMCU has inputs and outputs. Where the first input of the system is the DHT 11 sensor which is sense the humidity and temperature of the site. While the second input of the system is the LDR sensor which sense the light that comes from the laser. This sensor will not work when the sand enters the machine to sieve because the sand will interrupt the light that comes

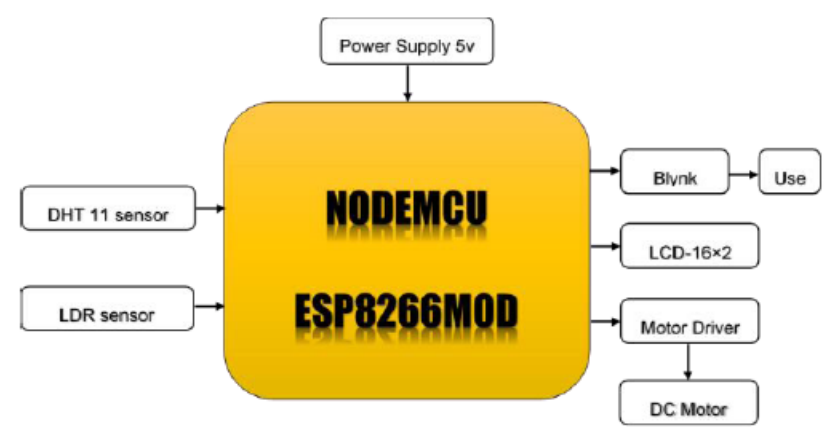

Figure 1: Block diagram of the system. from the laser. On the other side, the Blynk app considers an output because it will show the parameters of the speed of the motor, the humidity, and the temperature to the user. In addition to that, the LCD $-16 \times 2$ screen will appear the same parameters in the screen. While the motor driver will be responsible for moving the motor after getting the supply from the NodeMCU. This system works hand in hand to run the sand sieving machine.

\subsection{Mechanical Design}

The Figure 2 shows the sand sieving machine and its main parts. Where the machine was designed using SolidWork program [10]. The major purpose of the machine is to sieve the sand and make it free from impurities like grain and stones. The machine consists of a frame, wheels, channel, sieving mesh, connecting rod, belt drive, belt link, and motor. Where the frame used to support the body of the machine. The wheels are used as a support for the sieving mesh to move in the horizontal movement forward and backward [11]. While the channel uses allows the pure sand to flow in a specific place. The sieving mesh uses to filter the sand from impurities [12]. This mesh has different size holes, the use of each size comes as needed for the degree of purity of the sand from the impurities. As well as, the connecting rod used to move the sieving mesh after receiving the motion from the shaft. While the belt drive moved by the belt link after receiving the motion from the motor. Finally, the motor is responsible for the movement of the machine as a whole.

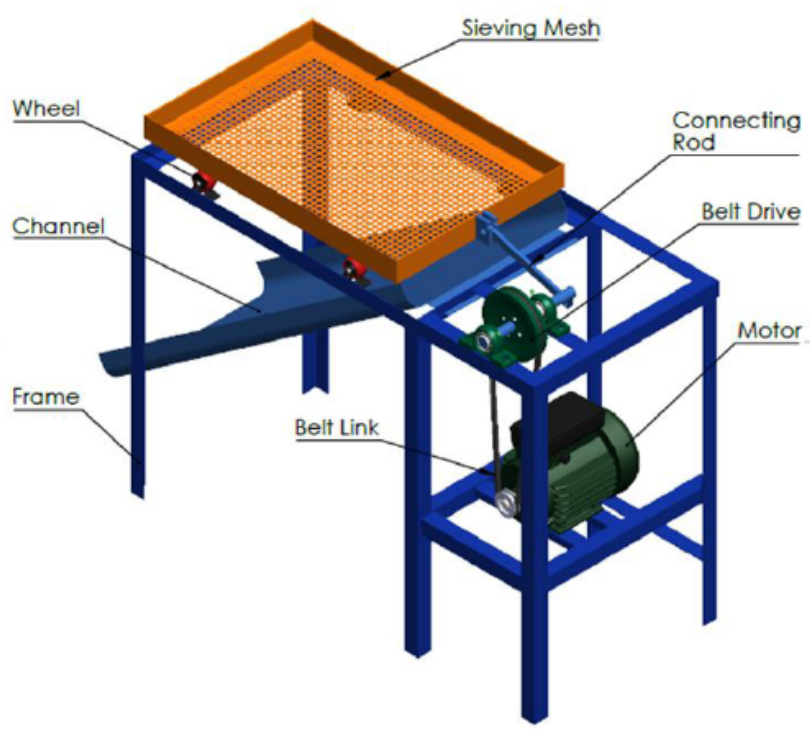

Figure 2: Sand sieving machine parts. 


\subsection{Circuit Design}

The circuit design shown in Figure 3 contains the connection of each electronic component with the NodeMCU through the breadboard terminals. Where it shows the correct location for all the inputs and outputs. Usually, the circuit design gives the user overview of the connections of the system. Where it helps to connect the components realistically without making mistakes. It is also considered an essential resource before implementing any programming for any microcontroller. In this circuit, the $12 \mathrm{v}$ power is considered as an adapter to supply the system. Where it connects directly to the motor driver for moving the DC motor. Where the DC motor used to cause the motion of the sieving mesh. In addition, the circuit showed the connection of the LCD-16×2 screen that displays the temperature and humidity values after it sense by the DHT 11 sensor. As shown in the circuit the LDR sensor and the laser must be opposite to each other to allow the sensor to receive the laser light.

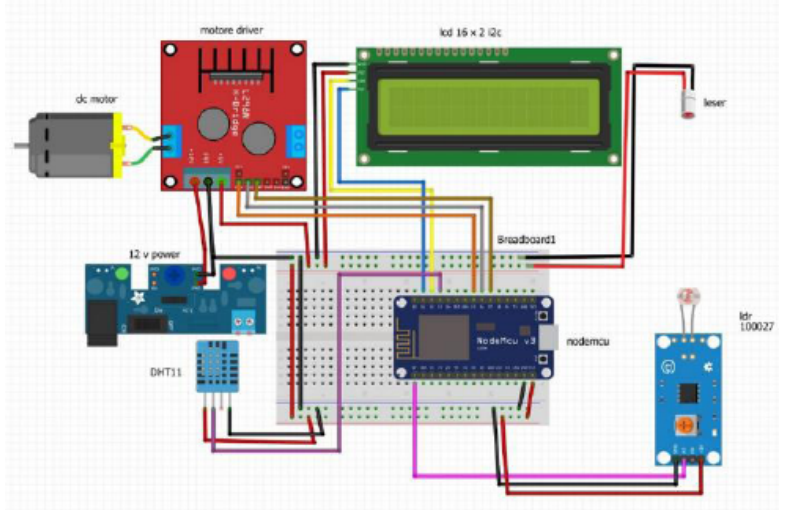

Figure 3: Circuit design of the system.

\subsection{Working Principle}

The working principle of the machine is divided into automatic working and manual work. That depends on the quantity of sand that want to sieve. Where the motor starts for the first time in the 30 s to sieve the sand. If there is any quantity of sand remaining, the motor will be controlled manually through the Blynk app to start again. When the operation is complete, the off button on the Blynk app will be pressed to terminate it. The temperature and humidity of the site will be displayed in three places as a result of this operation: first in the Blynk app, then in the LCD-162 screen, and finally in the serial sheet of the Arduino IED program. The operation in detail will be, the user will open the Blynk app to set the speed of the motor and check both the temperature and humidity. Then the NodeMCU will

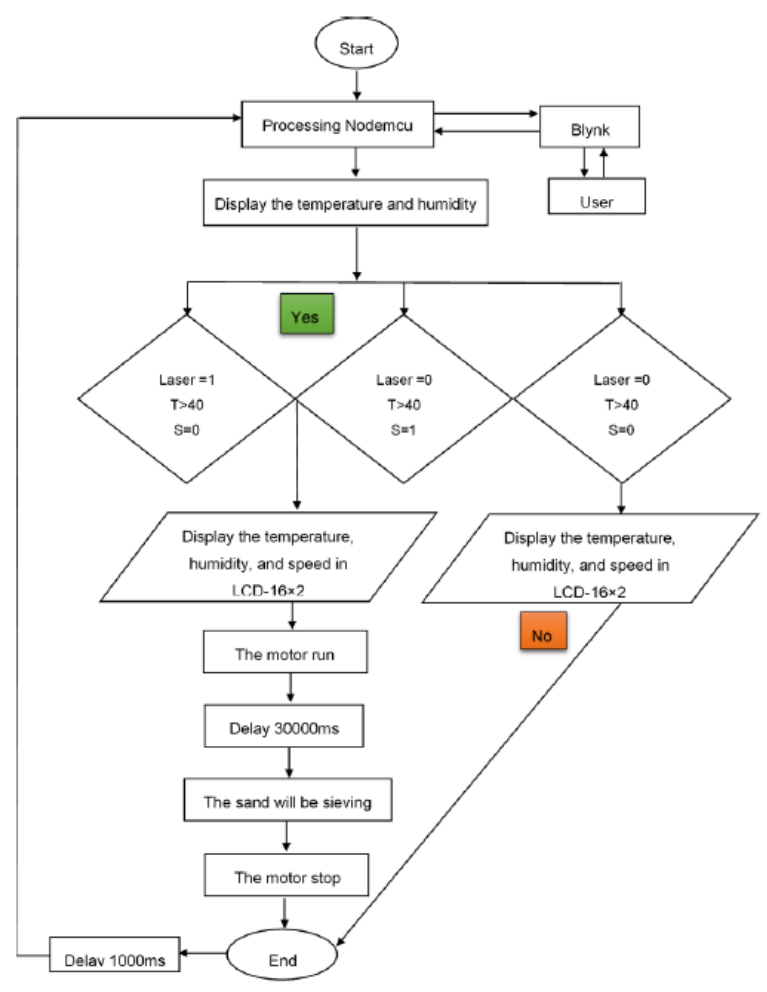

Figure 4: Flowchart of the system.

be connected with the Blynk app through a Wi-Fi network. The USB wire will link the computer with NodeMCU to download the program. After downloading the program, the USB wire will be removed. Hence, the adapter with $12 \mathrm{v}$ will supply the machine to work. The DHT 11 sensor will work to sense the temperature and humidity in the site and display it in the LCD $-16 \times 2$ screen. Also, the LDR sensor will receive light from the laser. That means the motor still not working because there is no sand cut off the laser light from the sensor. While the $D C$ motor is already receiving power from the motor driver to move. All of these operations have resulted in the machine being ready to use. The sand is now being poured into the sieve mesh. As a result, the amount of sand will cut off the laser light from the LDR sensor. Then the motor will start working according to the type of speed selected from the Blynk app. The Blynk app will show that the machine is working and the serial sheet will show that sand on the machine. This operation will repeat according to different speeds selected from the Blynk app and the amount of sand poured. The machine's goal is that when a large amount of sand is poured into the sieve mesh, the motor will start at a low or medium speed to sieve the sand. This will happen at 30 s, if there is any amount of sand still remaining in the sieve mesh the speed will shift to fast speed and the machine will operate manually through the Blynk app. The motor will run 
then will stop for $1 \mathrm{~s}$ to check if there is any sand remaining in the sieve mesh. If the quantity finished the off button will be pressed from the Blynk app to stop the operation. In this way, the power consumed will be saved and no waste will occur.

\subsection{Algorithm of the System}

Step1: start.

Step2: Processing NodeMCU.

Step3: The user will open the Blynk program to see a temperature and humidity.

Step4: The temperature and humidity will display in the LCD-16×2.

Step5: Laser will check the condition of for loop (= $1, T>40, S=0$ ) if it is Yes go to step 8, otherwise go to step 6.

Step6: Laser will check the second condition of for loop $(=0, T>40, S=1)$ if it is Yes go to step 8 , otherwise go to step 7 .

Step7: Laser will check the condition of for loop (= $0, T>40, S=0$ ) if it is No go to step13.

Step8: The temperature and humidity, and speed will display in the LCD $-16 \times 2$.

Step9: Motor will run.

Step10: The motor delay for 30s until the operation finish.

Step11: The sand will be sieving.

Step12: The motor will stop.

Step13: End.

Step 14: The motor delay for $1 \mathrm{~s}$ to be ready for the second operation and repeat the process again.

\section{RESULTS AND DISCUSSION}

The result and discussion will contain the calculations of the main parameters which is speed, mass, and time. In addition, it will show the work done for the machine and the kinetic energy during the motion. Moreover, it will include the discussion of the result in details. Result obtained from the Blynk App for speeds when motor run for different speed. A sample calculation for speed $100 \mathrm{rpm}$ is shown below

\subsection{Sample Calculation}

- Convert from rpm to $\mathrm{mls}$

$$
\begin{gathered}
v_{1}=\frac{2 \pi}{60} \times r p m \\
v_{1}=\frac{2 \pi}{60} \times 100=10.47 \mathrm{~m} / \mathrm{s}
\end{gathered}
$$

- Convert from g to $\mathrm{kg}$

$$
\begin{gathered}
1 \mathrm{~g}=0.001 \mathrm{~kg} \\
\therefore 90 \mathrm{~g}=0.09 \mathrm{~kg}
\end{gathered}
$$

Which is the mass will be fixed for all speeds

- $\quad$ Find the distance $(d)$ where $t_{1}=$

$$
\begin{gathered}
32 s \& v_{1}=10.47 \mathrm{~m} / \mathrm{s} \\
d_{1}=v_{1} \times t_{1} \\
d_{1}=10.47 \times 32=335.04 \mathrm{~m}
\end{gathered}
$$

- $\quad$ Find the force $(F)$ where $m=$

$0.09 \mathrm{~kg} \& a=$

$9.81 \mathrm{~m} /$

$s^{2}$ which accleration due to gravity

$$
\begin{gathered}
F=m \times a \\
F=0.09 \times 9.81=0.883 \mathrm{~N}
\end{gathered}
$$

Which is the mass will be fixed for all speeds

- $\quad$ Find the work done $(W)$ where $F=$

$$
\begin{gathered}
0.883 N \& d_{1}=335.04 m \\
W=F \times d_{1} \\
W=0.883 \times 335.04=295.84 J
\end{gathered}
$$

- $\quad$ Find the Kinetic Energy $(K E)$ where $m=$

$$
\begin{gathered}
0.09 \mathrm{~kg} \& v_{1}=10.47 \mathrm{~m} / \mathrm{s} \\
K E=\frac{1}{2} \times m \times v_{1}^{2} \\
K E=\frac{1}{2} \times 0.09 \times(10.47)^{2}=4.9 \approx 5 J
\end{gathered}
$$




\subsection{Calculations Results}

Table 1: Summarize of the Calculations

\begin{tabular}{|c|c|c|c|}
\hline \multirow{2}{*}{ Parameters } & \multicolumn{3}{|c|}{ Speeds } \\
\cline { 2 - 4 } & $\mathbf{1 0 0} \mathbf{~ r p m}$ & $\mathbf{1 7 0} \mathbf{~ r p m}$ & $\mathbf{2 2 5} \mathbf{~ r p m}$ \\
\hline \hline Speed in $\mathbf{~} / \mathbf{s}(\mathbf{v})$ & $10.47 \mathrm{~m} / \mathrm{s}$ & $17.8 \mathrm{~m} / \mathrm{s}$ & $23.56 \mathrm{~m} / \mathbf{s}$ \\
\hline Mass (m) & $0.09 \mathrm{~kg}$ & $0.09 \mathrm{~kg}$ & $0.09 \mathrm{~kg}$ \\
\hline Distance (d) & $335.04 \mathrm{~m}$ & $213.6 \mathrm{~m}$ & $141.36 \mathrm{~m}$ \\
\hline Force (F) & $0.883 \mathrm{n}$ & $0.883 \mathrm{n}$ & $0.883 \mathrm{n}$ \\
\hline Work done (W) & $295.84 \mathrm{j}$ & $188.61 \mathrm{j}$ & $124.82 \mathrm{j}$ \\
\hline Kinetic Energy (KE) & $5 \mathrm{j}$ & $14.25 \mathrm{j}$ & $24.97 \mathrm{j}$ \\
\hline
\end{tabular}



Figure 5: Graph of the relation between speed and time.

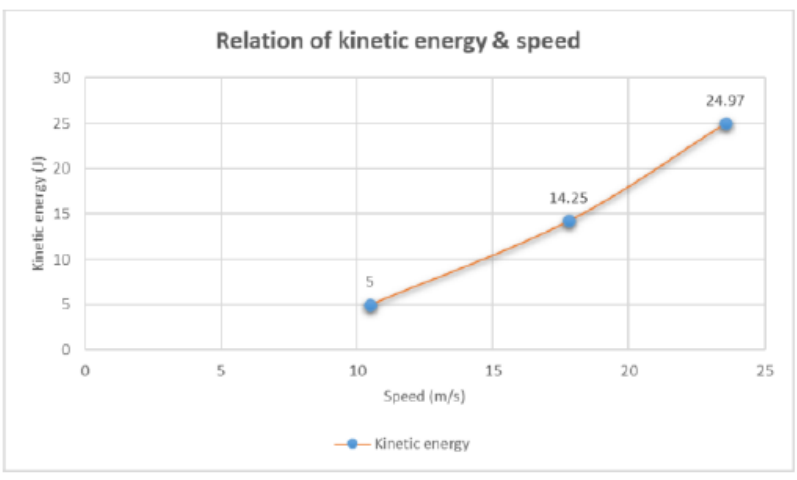

Figure 6: Graph of the relation between kinetic energy and speed.

According to the result the study discusses several point starting from the Blynk software observation and ending with the graphs Figure $\mathbf{5}$ and Figure $\mathbf{6}$ obtained from the calculations. Where the Blynk monitor both the temperature and humidity when the motor run the operation. The purpose of this monitoring process is to tell the user or worker that the site is safe when the machine was running. Because, the temperature set under specific condition, if the temperature in the site more than $40 \mathrm{C}$ the machine will not work. That to save the worker from high temperature and save the machine from damage. In addition to that, the app used to select the level of speed according to the quantity of sand that poured in the sieve mesh. The speeds vary from slow to medium to fast. Hence the slow speed equal to $100 \mathrm{rpm}$, the medium speed equal to $170 \mathrm{rpm}$, and the fast speed equal to $225 \mathrm{rpm}$. Moreover, the Blynk app offers to the use manual button to on \& off the machine if the quantity is more and takes more than 30 s to finished sieving all the quantity. In other side, the time was taken by using the stopwatch to monitor how much the $90 \mathrm{~g}$ of sand to sieve through different level of speeds. Hence, obtained that the slow speed takes more time to sieve whole amount of sand through the sieving mesh. Where it takes around of 32s to sieve the sand which means the manual button was pressed to finish whole the amount. While obtained that the medium speed takes $12 \mathrm{~s}$ to sieve the sand through the sand mesh. This is quite an appropriate time to finish the amount during 30s. However, the fast speed finished the operation in earlier time which around $6 \mathrm{~s}$. These differences in the sieving process refers that the size of the impurities varies every time of the amount of sand is placed on the mesh. In the other hand, the interface of the serial sheet of the Arduino IDE software a proved the operation of the machine when the motor was running. Where it shows the situation of the machine when the sand crossing the sieving mesh of the machine. Usually, the serial sheet displays the update of the machine through the temperature \& humidity values in the site, the condition of either sand poured or not, the condition of either the motor on or off, and the manual button either pressed or not. While, when the motor running it only shows the motor on and the sand crossed the sieving mesh. Beside all of that, the calculations process of the speed was done through different sides. First, the converting all of the speeds from $\mathrm{rpm}$ to $\mathrm{m} / \mathrm{s}$. Where the slow speed equal to $10.47 \mathrm{~m} / \mathrm{s}$, the medium speed equal to $17,8 \mathrm{~m} / \mathrm{s}$, and the fast speed equal to $23.56 \mathrm{~m} / \mathrm{s}$. Second, the converting of mass from $\mathrm{g}$ to $\mathrm{kg}$ where the amount of sand that used equal to $0.09 \mathrm{~kg}$. Third, calculating the distance of each speed after getting the time and speed in $\mathrm{m} / \mathrm{s}$. Where the distance of the slow speed equal to $335.04 \mathrm{~m}$, the distance of the medium speed equal to $213.6 \mathrm{~m}$, and the distance of the fast speed equal to $141.36 \mathrm{~m}$. Fourth, the force acting on the machine according the mass of the amount of sand that poured in the machine and the acceleration due to gravity. Where the force equal to $0.883 \mathrm{~N}$ and it's fixed for all speeds. Fifth, the work done on the machine due to the force and distance per each speed. Where the work done of the slow speed equal to $295.84 \mathrm{~J}$, the 
work done of the medium speed equal to $188.61 \mathrm{~J}$, and the work done of the fast speed equal to $124.82 \mathrm{~J}$. Resulting from that when the speed increases the work done decrease there is inverse proportional between them. Finally, the kinetic energy off the motion of machine was calculated according to the different speeds. Where the kinetic energy of the slow speed equal to $5 \mathrm{~J}$, the kinetic energy of the medium speed equal to $14.25 \mathrm{~J}$, and the kinetic energy of the fast speed equal to $24.97 \mathrm{~J}$. All of this result was summarized in the Table 1 . The last section of the result contains the graph relation. Where it shows the speed relation with both time and kinetic energy. Hence, the first graph showed the reverse relation between the time and the speed. When the speed increases, the time was decrease. So, the machine during the fast speed it will take less time to execute the operation and sieve the sand. While in the second graph showed the forward relation between the speed and kinetic energy. When the speed increases, the kinetic energy was increase. So, the machine during the fast speed it will take more kinetic energy to execute the operation and sieve the sand.

\section{CONCLUSION}

The continuity of the study lies in replacing the traditional methods of sand separation with modern methods using sensors to reduce the time and effort on workers. Besides, the sand is separated by changing the mesh size. According to what is commensurate with the sizes of sand and the amount used. By NodeMCU, sensors provide protect the power consumption of the motor from a continuous operation. Where the DC motor operates after the sensors respond to sand entering the machine. Therefore, that it converts the energy into a back-and-forth sliding motion of the sieving mesh. Hence, the sand is separated automatically without human intervention.

\section{REFERENCE}

[1] AL. BLAIR, "The Importance Sand and Gravel in Our Lives," 2021. https://alblairconstruction.com/importance-of-sandand-gravel/ (accessed Aug. 30, 2021).

[2] AK. Nachimuthu, S. Ragunath, S. Mohanavelan, PPN. Han, and S. Mariraj, "Design and fabrication of horizontal sieving machine," Int. Res. J Multidiscip Sci Technol 2016; pp: 103106.

[3] SV. Hapsenkar et al., "Design and Fabrication of Industrial Sand Screening Machine for Green Sand," Int. J. Res. Eng. Sci. Manag 2020; 3(11): 16-20.

[4] DB. Wiki, "Sand," 2020 https://www.designingbuildings.co.uk/wiki/Sand (accessed Aug. 31, 2021)

[5] P. Irish, "The Importance of Getting the Right Quality of Sand," 2020 https://www.dreamlandsdesign.com/importance-getting-rightquality-of-sand/ (accessed Aug. 31, 2021).

[6] G. Kurnia, B. Yulianto, J. Jamari, and AP. Bayuseno, "Evaluation in Conceptual Design of Human Powered Sand Sieving Machine," E3S Web Conf 2019; 125(201) 9: 0-4. https://doi.org/10.1051/e3sconf/201912503001

[7] A. Boy et al., "Design and Fabrication of Inclinable Trommel Sand Sieve Machine," Int. Res. J. Eng. Technol 2017; 7(5): 2-10.

[8] Wikipedia, "Sieve," Wikimedia Foundation, Inc, 2021 https://en.wikipedia.org/wiki/Sieve (accessed Aug. 31, 2021).

[9] AU. Tigadikar, A. Aranhunshi, and S. Patil, "Design and Fabrication of Semi Automated Solar Powered Sand Sieving Machine," Int. J. Eng. Res. Technol 2019; 8(11): 856-858.

[10] PR. Gajbhiye, R. Khode, P. Sukhadeve, and V. Chaple, "Design and Fabrication of Automatically Driven Sand Sieving," 8International J. Eng. Res. Technol 2019; 8(11): 856-858. [Online]. Available: https://www.ijert.org.

[11] VP. Duriraj and J. Manikandan, "Design and Fabrication of Sieveing Machine," 2017; 116(19): 447-451.

[12] M. Ramrao, B. Mahesh, B. Renuka, B. Rampal, and M Rajesh, "Design and Fabrication of Development of Sand filter and Separator Pedal Powered," Int. J. Emerg. Technol. Eng. Res 2018; 6(3): 128-132. 